05,12

\title{
Ферромагнитный резонанс во взаимодействующих магнитных микрополосках
}

\author{
(C) Р.В. Горев ${ }^{1}$, Е.В. Скороходов ${ }^{1,2}$, В.Л. Миронов ${ }^{1,2}$ \\ ${ }^{1}$ Институт фозики микроструктур РАН, \\ Нижний Новгород, Россия \\ ${ }^{2}$ Нижегородский государственный университет им. Н.И. Лобачевского, \\ Нижний Новгород, Россия \\ E-mail: GorevRV@ipm.sci-nnov.ru
}

\begin{abstract}
Приводятся результаты микромагнитного моделирования вынужденных колебаний намагниченности в системе двух взаимодействующих микрополосок, расположенных под углом друг к другу. Изучены спектры ферромагнитного резонанса и модовый состав резонансных колебаний системы в условиях магнитостатического и обменного взаимодействий между полосками. Показано, что магнитостатическое взаимодействие приводит к возможности возбуждения синфазных и противофазных связанных колебаний намагниченности полосок. В системах обменно-связанных микрополосок реализуются интенсивные резонансы, обусловленные колебаниями доменных стенок. Обсуждается перестройка спектра и модового состава резонансных колебаний в зависимости от равновесной конфигурации намагниченности системы, а также условия возбуждения колебаний различного типа в зависимости от направления магнитного СВЧ-поля.
\end{abstract}

Работа выполнена при поддержке Российского научного фонда (проект РНФ № 16-12-10254).

\section{1. Введение}

Ферромагнитные наноструктуры традиционно привлекают внимание многих исследовательских групп в связи с перспективностью их применения в устройствах хранения информации и приборах СВЧ-наноэлектроники [13]. В последнее время в связи с развитием современных методов нанолитографии появилась возможность создания сверхплотных планарных структур с характерными размерами элементов порядка $100 \mathrm{~nm}$ и расстояниями между элементами вплоть до $10 \mathrm{~nm}$ [4-6]. В таких системах становятся существенными эффекты магнитостатического и обменного взаимодействий. В частности, большое число работ посвящено исследованию влияния размерных эффектов на СВЧ-свойства ферромагнитных наносистем [7-17].

Одним из основных методов теоретического исследования СВЧ-свойств ферромагнитных наноструктур является моделирование, основанное на численном решении уравнения Ландау-Лифшица. В большинстве работ спектр ферромагнитного резонанса рассчитывается с помощью Фурье-анализа релаксационных колебаний, возбуждаемых импульсным магнитным полем [14,15]. Такой метод позволяет быстро рассчитать значения собственных частот исследуемой системы, однако не позволяет получить информацию об эффективности возбуждения той или иной моды колебаний внешним переменным полем. В настоящей работе исследовано влияние обменного и магнитостатического взаимодействий на СВЧсвойства систем с равновесным неколлинеарным распределением магнитного момента. Проанализировано влияние равновесной конфигурации намагниченности и направления магнитного СВЧ-поля на модовый состав спектра ферромагнитного резонанса (ФМР). С практической точки зрения интерес к данному направлению исследований обусловлен перспективами применения магнитных наноструктур для создания перестраиваемых резонаторов и пассивных фильтров гигагерцевого диапазона частот $[18,19]$.

\section{2. Методика расчетов}

Методом численного моделирования исследовались спектры ФМР и модовый состав колебаний намагниченности в системах, состоящих из двух взаимодействующих полосок пермаллоя $\mathrm{Ni}_{80} \mathrm{Fe}_{20}$. Моделирование проводилось на основе численного решения уравнения Ландау-Лифшица-Гильберта для намагниченности образца с использованием стандартного пакета Object Oriented Micromagnetic Framework [20]. В расчетах использовались следующие параметры $\mathrm{Ni}_{80} \mathrm{Fe}_{20}$ : намагниченность насыщения $8 \cdot 10^{5} \mathrm{~A} / \mathrm{m}$, обменная константа $8.4 \cdot 10^{-12} \mathrm{~J} / \mathrm{m}$, константа диссипации 0.01 , кристаллографическая анизотропия не учитывалась. Размер ячейки составлял $10 \times 10 \times 30 \mathrm{~nm}$, что является достаточным для описания неоднородных колебаний намагниченности в частотном диапазоне, который может быть экспериментально реализован $(1-20 \mathrm{GHz})$. Микрополоски имели характерный размер $2000 \times 500 \times 30 \mathrm{~nm}$. При моделировании колебаний намагниченности первоначально система приводилась в состояние равновесия, после чего к ней прикладывалось переменное магнитное поле и регистрировалась амплитуда установившихся колебаний, пропорциональная поглощенной мощности СВЧ-излучения. Частота возбуждающего СВЧ-поля изменялась в диапазоне от 0 до $14 \mathrm{GHz}$ с шагом $0.1 \mathrm{GHz}$. Внешнее постоянное магнитное поле отсутствовало. Для анализа модового состава резонансов рассчитывались временны́е реализации пространственных распределений колебаний $z$-компоненты намагниченности. 


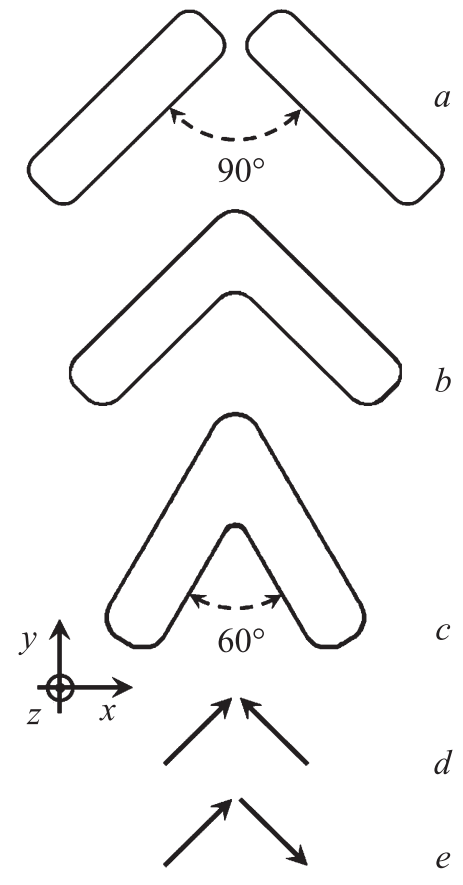

Рис. 1. Системы взаимодействующих микрополосок. $a-$ система отдельных полосок, расположенных под углом $90^{\circ}$ (система I). $b-$ связанные полоски, расположенные под углом $90^{\circ}$ (система II). $c$ - связанные полоски, расположенные под углом $60^{\circ}$ (система III), $d-$ состояние магнитных моментов полосок $\Gamma-\Gamma . e-$ состояние $\Gamma-\mathrm{X}$.

\section{3. Результаты и обсуждение}

Было проведено исследование трех систем. Первая система (система I, рис. 1,a) представляла собой две частицы, угол между длинными осями которых составлял $90^{\circ}$, зазор между краями частиц равнялся $100 \mathrm{~nm}$. Во второй системе (система II, рис. 1,b) угол между осями частиц также был равен $90^{\circ}$, но частицы были соединены между собой, т.е. они были обменно связаны. В третьей системе (система III, рис. 1,c) угол между осями частиц равнялся $60^{\circ}$, частицы также были соединены. Каждая из этих систем может находиться в двух стабильных состояниях, которые соответствуют конфигурациям „голова к голове“ $(\Gamma-\Gamma)($ рис. $1, d)$ или „голова к хвосту“ $(\Gamma-\mathrm{X})$ (рис. $1, e)$. Переходы системы из одного состояния в другое осуществляются под действием импульса внешнего магнитного поля (характерная величина перемагничивающего поля $\sim 200 \mathrm{Oe})$.

Взаимодействие в исследуемых системах приводит к возникновению коллективных колебаний. При этом, меняя способ возбуждения колебаний (направление переменного СВЧ-поля), можно управлять спектром поглощения СВЧ, так как интенсивность различных мод зависит от условий возбуждения. В частности, в рассматриваемых системах возникают моды квазиоднородных колебаний: квазиоптические (противофазные связанные колебания) и квазиакустические (синфазные связанные колебания). Каждую из них в отдельности можно воз- будить, если прикладывать переменное поле по осям $x$ или y (направление осей показано на рис. 1).

Спектры системы I приведены на рис. 2. Интенсивные пики в данных спектрах соответствуют возбуждению квазиоптической и квазиакустической мод. Для конфигурации $Г-Г$ квазиакустическая мода возбуждается при приложении переменного поля по оси $x$ (спектр показан сплошной линией на рис. 2, $a$ ), а квазиоптическая - при приложении переменного поля по оси $y$ (спектр показан штриховой линией на рис. 2,a). Для конфигурации Г-Г квазиакустическая мода возбуждается при приложении переменного поля по оси $y$ (штриховая линия на рис. $2, b)$, а квазиоптическая - при приложении переменного поля по оси $x$ (сплошная линия на рис. $2, b$ ).

В качестве примера на рис. 3 приведены пространственные распределения амплитуды колебаний в квази-
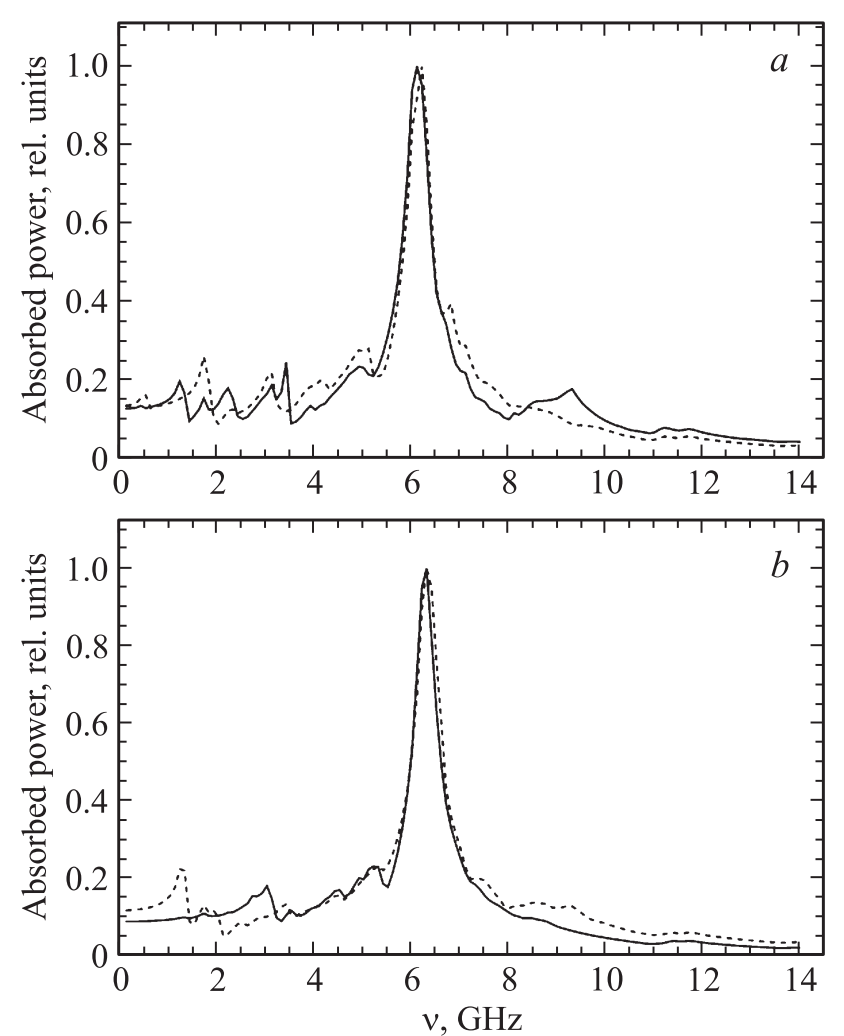

Рис. 2. Модельные спектры системы I для конфигурации $\Gamma-\Gamma(a)$ и $\Gamma-\mathrm{X}(b)$. Сплошной линией показаны спектры, полученные при приложении переменного поля по оси $x$, штриховой - при приложении поля по оси $y$.

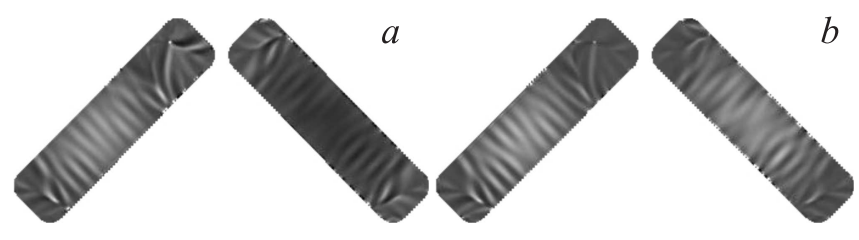

Рис. 3. Пространственные распределения амплитуды колебаний $z$-проекции намагниченности в системе I для конфигурации $\Gamma-\Gamma$, соответствующие квазиоптической $(a)$ и квазиакустической $(b)$ модам. 
оптической и квазиакустической модах для конфигурации Г-Г системы І. Из рис. 3 видно, что резонансные колебания представляют собой суперпозицию квазиоднородной моды и мод продольного спин-волнового резонанса. Это обусловлено немонотонностью дисперсионной характеристики спиновых волн, распространяющихся вдоль длинной оси полоски, что ведет к вырождению частоты, т.е. одному значению частоты соответствуют две спин-волновые моды [14]. Однако спин-волновые моды с высокой пространственной частотой вносят слабый вклад в интенсивность резонансных пиков из-за малости интеграла перекрытия в выражении для коэффициентов возбуждения мод [21]. Остальные малоинтенсивные пики связаны с резонансными колебаниями небольших неоднородных областей в распределении намагниченности микрополосок. Перестройка частоты основного резонанса системы I при смене конфигурации намагниченности не превышает $0.2 \mathrm{GHz}$.

Спектры системы II представлены на рис. 4. Резонансные колебания системы II, отвечающие основному пику, аналогичны квазиоднородным модам системы I. Перестройка частоты основного пика при смене конфигурации намагниченности для системы II достигает значения $0.8 \mathrm{GHz}$. Однако объединение двух частиц в один „уголок“ приводит к тому, что в данной системе возникают две доменные стенки в распределении намагниченности, соответствующем состоянию Г-Г, и одна
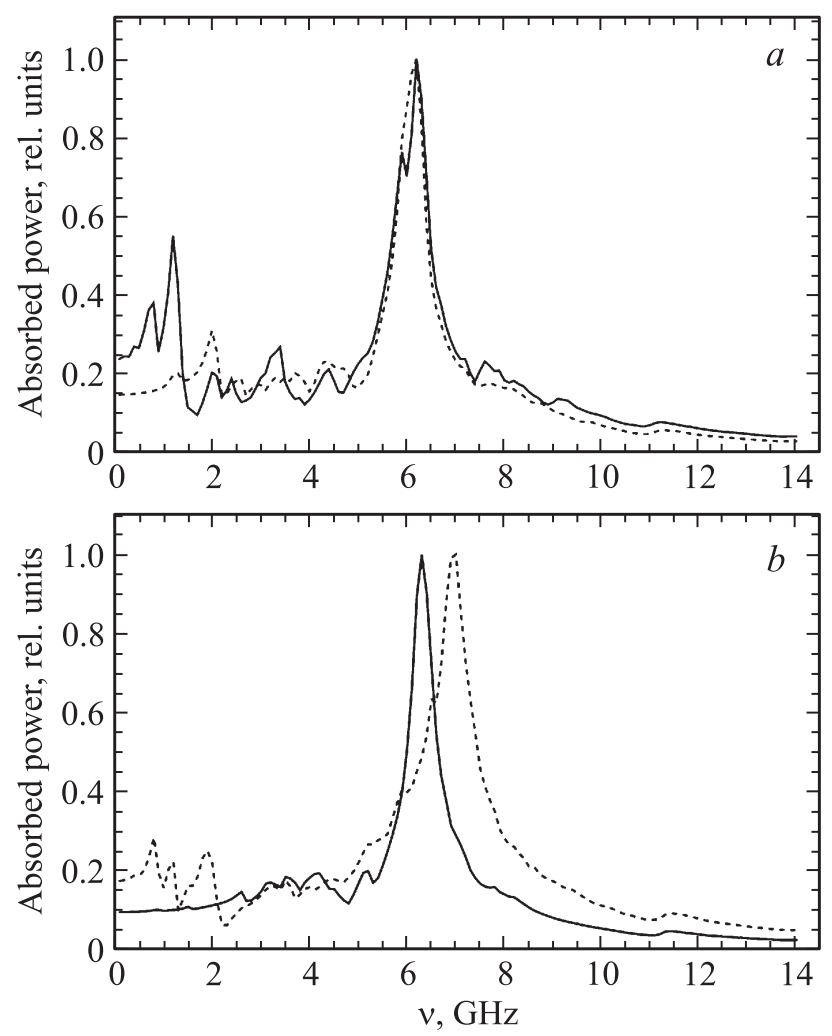

Рис. 4. Модельные спектры системы II для конфигурации $\Gamma-\Gamma(a)$ и $\Gamma-\mathrm{X}(b)$. Сплошной линией показаны спектры, полученные при приложении переменного поля по оси $x$, штриховой - при приложении поля по оси $y$.

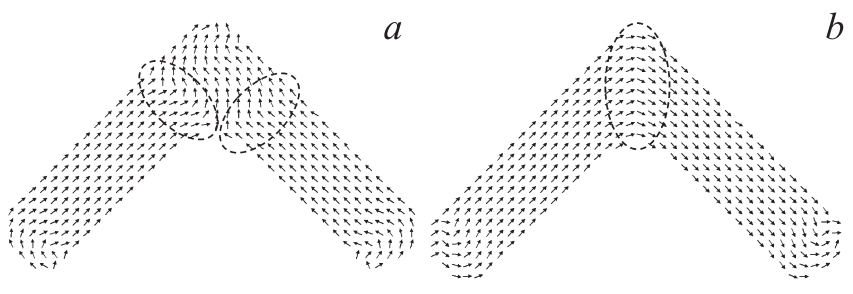

Рис. 5. Равновесные распределения намагниченности в системе II для конфигураций $\Gamma-\Gamma(a)$ и $\Gamma-\mathrm{X}(b)$. Области доменных стенок выделены овалами.

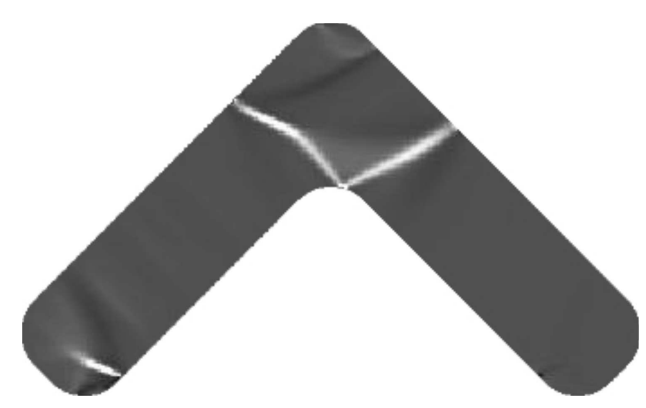

Рис. 6. Пространственное распределение амплитуды резонансных колебаний $z$-компоненты намагниченности при конфигурации $\Gamma-\Gamma$ для системы II на частоте $v=1.2 \mathrm{GHz}$. Переменное поле приложено по оси $x$.

доменная стенка в состоянии $\Gamma-\mathrm{X}$ (рис. 5). Области доменных стенок являются дополнительными осцилляторами, которые находятся во взаимодействии с другими частями системы, поэтому в спектрах появляются соответствующие им дополнительные резонансные пики.

В системе II наличие доменных стенок в конфигурации $\Gamma-\Gamma$ (рис. $5, a)$ приводит к возникновению в спектре достаточно интенсивного пика на частоте $v=1.2 \mathrm{GHz}$ при приложении поля по оси $x$. Распределение амплитуд колебаний намагниченности, соответствующее данному резонансу, показано на рис. 6. Можно также заметить, что в этом случае присутствует резонанс вблизи края полоски, обусловленный неоднородностью намагниченности в этой области. Отметим, что при приложении переменного поля вдоль оси $y$ данный резонанс возбуждается менее эффективно (см. спектр на рис. 4,a). Это связано с тем, что распределение намагниченности в доменных стенках для данной конфигурации имеет преимущественное направление вдоль оси $y$, а следовательно, приложение СВЧ-поля вдоль оси $x$ ведет к более эффективному возбуждению резонанса.

Для конфигурации $\Gamma-\mathrm{X}$ и направления переменного поля по оси $y$ также наблюдается заметный резонанс, соответствующий основному колебанию доменной стенки (рис. 7). При этом переменное поле, направленное вдоль оси $x$, практически не возбуждает колебаний доменной стенки, что, как и в предыдущем случае, связано с особенностями в распределении намагниченности (намагниченность направлена преимущественно вдоль оси $x$ (рис. $5, b))$. 


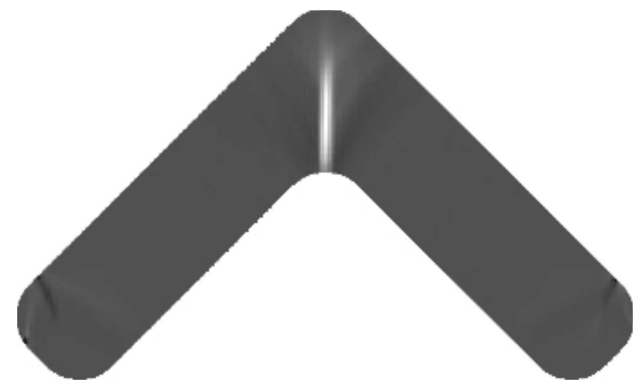

Pис. 7. Пространственное распределение амплитуды колебаний $z$-компоненты намагниченности при конфигурации $\Gamma-\mathrm{X}$ для системы II на частоте $v=1.9 \mathrm{GHz}$. Переменное поле было приложено по оси $y$.
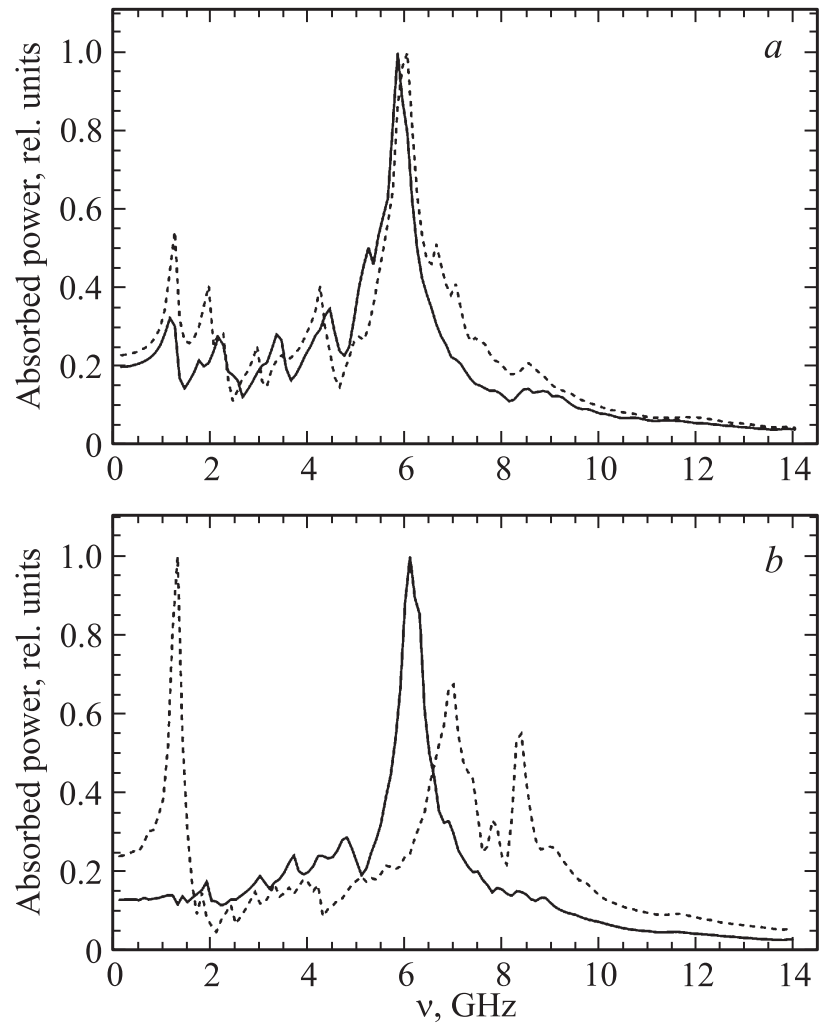

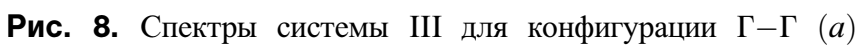
и $\Gamma-\mathrm{X}(b)$. Сплошной линией показаны спектры, полученные при приложении переменного поля по оси $x$, штриховой при приложении поля по оси $y$.

Спектры системы III представлены на рис. 8. Как и для системы II, в спектрах присутствуют квазиоднородные моды и моды, связанные с колебаниями доменных стенок. Распределения намагниченности для конфигураций $\Gamma-\Gamma$ и $\Gamma-\mathrm{X} \mathrm{показаны} \mathrm{на} \mathrm{рис.} 9$.

Для конфигурации $\Gamma-\Gamma$, также как и для системы II, присутствует достаточно интенсивный пик на частоте $v=1.2 \mathrm{GHz}$ (рис. $10, a)$, соответствующий резонансу, связанному с доменной стенкой. Данный резонанс хорошо возбуждается переменным полем, направленным как вдоль оси $x$, так и вдоль оси $y$. Это связано с тем, что равновесная конфигурация намагниченности в доменных стенках для системы III не имеет преимущественного направления вдоль какой-либо из осей. Остальные пики, представленные на рис. $8, a$, соответствуют спинволновым резонансам. На рис. $10, b$ показано пространственное распределение колебаний одной из мод, соответствующей спин-волновому резонансу на частоте $v=4.4 \mathrm{GHz}$.

Для конфигурации $\Gamma-\mathrm{X}$ и направления переменного поля по оси $x$ наблюдается несколько интенсивных резонансных пиков (рис. $8, b$ ). Кроме квазиоднородной моды, имеющей частоту $v=6.1 \mathrm{GHz}$, присутствуют пики, связанные с колебаниями доменной стенки на частотах $v=1.3$ и $8.4 \mathrm{GHz}$. Соответствующие распределения колебаний представлены на рис. 11. Резонанс на частоте $v=1.3 \mathrm{GHz}$ аналогичен резонансу в системе II на ча-

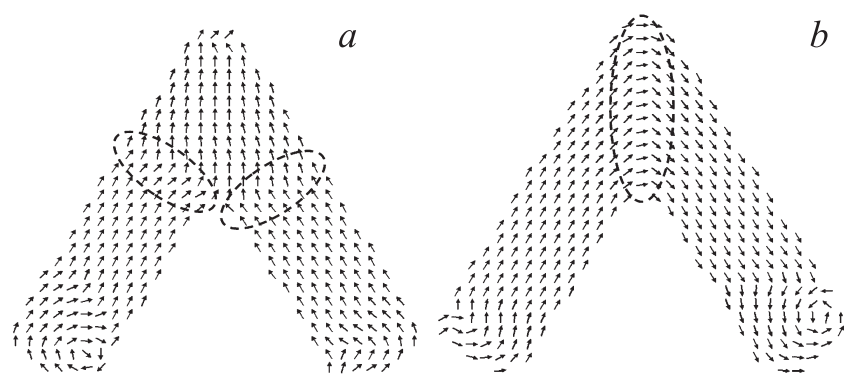

Рис. 9. Равновесные распределения намагниченности в системе III для конфигураций $\Gamma-\Gamma(a)$ и $\Gamma-\mathrm{X}(b)$.
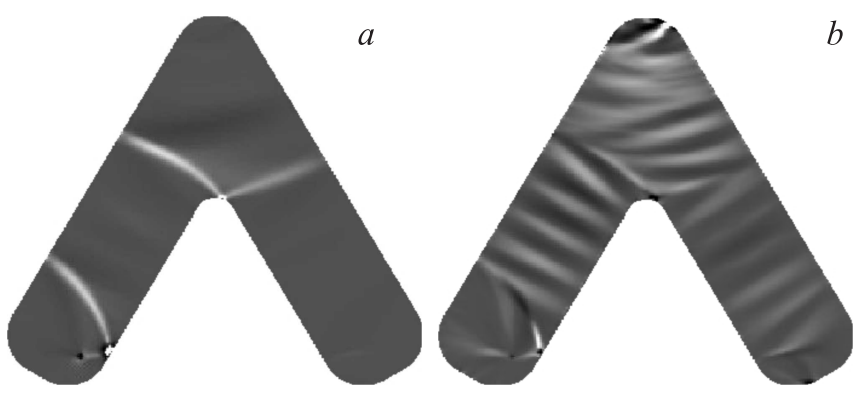

Рис. 10. Пространственные распределения амплитуд колебаний $z$-компоненты намагниченности при конфигурации $\Gamma-\Gamma$ для системы III на частотах $v=1.2 \mathrm{GHz}(a)$ и $v=4.4 \mathrm{GHz}(b)$. Переменное поле приложено по оси $x$.
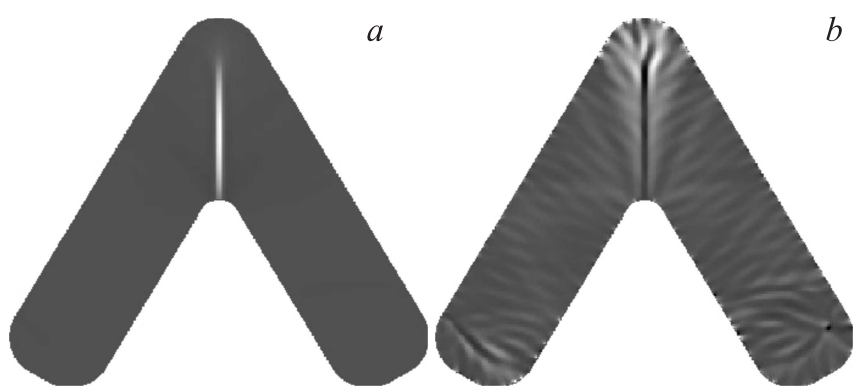

Рис. 11. Пространственные распределения амплитуд колебаний $z$-компоненты намагниченности при конфигурации $\Gamma-\mathrm{X}$ для системы III на частотах $v=1.3(a)$ и $v=8.4 \mathrm{GHz}(b)$. 
Резонансные частоты (в GHz) квазиоднородной моды для различных систем взаимодействующих полосок

\begin{tabular}{c|c|c|c}
\hline Конфигурация & I & II & III \\
\hline$\Gamma-Г$, поле по оси $x$ & 6.1 & 6.2 & 5.8 \\
$\Gamma-Г$, поле по оси $y$ & 6.2 & 6.2 & 6.0 \\
$\Gamma-\mathrm{X}$, поле по оси $x$ & 6.3 & 6.3 & 6.1 \\
$\Gamma-\mathrm{X}$, поле по оси $y$ & 6.3 & 7.0 & 7.0
\end{tabular}

стоте $v=1.9 \mathrm{GHz}$. Так же как и в системе II, данный резонанс возбуждается переменным полем, направленным вдоль оси $y$. Резонансные колебания намагниченности внутри доменной стенки можно описать количеством укладывающихся в ней полуволн аналогично тому, как это делается для однородно намагниченных микрополосок [14]. Пик, находящийся на частоте $v=8.4 \mathrm{GHz}$, отвечает колебанию доменной стенки с числом полуволн, равным трем (рис. $11, b$ ).

Для сравнения в таблице приведены значения частот квазиоднородных мод для всех изучаемых конфигураций. Видно, что наибольшие изменения в положении резонансных пиков наблюдаются для систем II и III, что связано с более сильным магнитостатическим взаимодействием между микрополосками.

Анализ полученных результатов показывает, что максимальное изменение частоты резонанса, соответствующего квазиоднородным модам, при изменении конфигурации намагниченности наблюдается в системе III и составляет $1 \mathrm{GHz}$. С другой стороны, положения резонансных пиков, соответствующих колебаниям доменной стенки, чувствительны к конфигурации намагниченности и ориентации системы относительно переменного магнитного поля. В частности, для системы III, находящейся в конфигурации $\Gamma-\mathrm{X}$, при направлении СВЧ-поля по оси $y$ наблюдается резонансный пик, связанный с колебаниями доменной стенки, интенсивность которого сравнима с интенсивностью пика, соответствующего квазиоднородной моде. Однако при приложении переменного поля по оси $x$ данный пик практически исчезает (рис. $8, b$ ). Это делает использование резонансных колебаний доменных стенок перспективным с точки зрения реализации перестраиваемых СВЧ-фильтров в диапазоне частот около $1 \mathrm{GHz}$.

\section{4. Заключение}

Таким образом, магнитостатическое взаимодействие приводит к перестройке спектра ФМР системы взаимодействующих микрополосок. Неколлинеарность распределения магнитного момента в изученных системах приводит к возможности возбуждения не только синфазных колебаний, но и противофазных связанных колебаний внешним однородным магнитным СВЧ-полем. В системах обменно-связанных микрополосок взаимодействие между частицами приводит к расщеплению мод квазиоднородной прецессии и появлению дополнительных пиков, обусловленных образованием доменных стенок. Характер распределений намагниченности в доменных стенках влияет как на положение соответствующих резонансных пиков, так и на эффективность возбуждения резонансов в зависимости от направления магнитного СВЧ-поля. Продемонстрировано, что спектр поглощения СВЧ-излучения данными системами существенно зависит от конфигурации намагниченности, угла между микрополосками и ориентации относительно переменного магнитного поля.

\section{Список литературы}

[1] S.S.P. Parkin, K.P. Rodhe, M.G. Samant, P.M. Rice, R.B. Beyers, R.E. Scheuerlein, E.J. O’Sallivan, S.L. Bruwn, J. Bucchigano, D.W. Abraham, Yu. Lu, M. Rooks, P.L. Trouilliud, R.A. Wanner, W.J. Callagher. J. Appl. Phys. 85, 5828 (1999).

[2] J.-G. Zhu. Proc. IEEE 96, 11, 1786 (2008).

[3] R.L. Stamps, S. Breitkreutz, J. Akerman, A.V. Chumak, Y. Otani, G.E.W. Bauer, J.-U. Thiele, M. Bowen, S.A. Majetich, M. Klaui, I.L. Prejbeanu, B. Dieny, N.M. Dempsey, B. Hillebrands. J. Phys. D 47, 333001 (2014).

[4] А.А. Фраерман. УФН 182, 12, 1345 (2012).

[5] C. Vieu, F. Carcenac, A. Pepin, Y. Chen, M. Mejias, A. Lebibv, L. Manin-Ferlazzo, L. Couraud, H. Launois. Appl. Surf. Sci. 164, 111 (2000).

[6] У. Моро. Микролитография. Мир, М. (1990). 1240 с.

[7] M. Zhu, R.D. McMichael. J. Appl. Phys. 109, 043904 (2011).

[8] Y. Nozaki, K. Tateishi, S. Taharazako, S. Yoshimura, K. Matsuyama. Appl. Phys. Lett. 92, 161903 (2008).

[9] X.H. Han, R.L. Liu, Q.F. Liu, J.B. Wang, T. Wang, F.S. Li. Physica B 405, 1172 (2010).

[10] A.O. Adeyeye, S. Jain. J. Appl. Phys. 109, $07 B 903$ (2011).

[11] В.А. Журавлев, А.А. Ошлаков. ФТТ 43, 11, 2025 (2001).

[12] В.Б. Гусева, А.Ф. Зацепин, В.А. Важенин, В. Schmidt, Н.В. Гаврилов, С.О. Чолах. ФТТ 47, 4, 650 (2005).

[13] Р.Б. Моргунов, А.И. Дмитриев, Г.И. Джардималиева, А.Д. Помогайло, А.С. Розенберг, Y. Tanimoto, M. Leonowicz, Е. Sowka. ФTТ 49, 8, 1436 (2007).

[14] Р.В. Горев, В.Л. Миронов, Е.В. Скороходов. Поверхность. Рентгеновские, синхротронные и нейтронные исследования 3, 37 (2016).

[15] R. Adam, Y. Khivintsev, R. Hertel, C. M. Schneider, A. Hutchison, R. Camely, Z. Celinski. J. Appl. Phys. 101, 09F516 (2007).

[16] M.P. Wismayer, B.W. Southern, X.L. Fan, Y.S. Gui, C.-M. Hu, R.E. Camley. Phys. Rev. B 85, 064411 (2012).

[17] V. Flovik, F. Macià, J.M. Hernàndez, R. Bručas, M. Hanson, E. Wahlström. Phys. Rev. B 92, 104406 (2015).

[18] B.K. Kuanr, R. Lopusnil, L.M. Malkinski, M. Wenger, M. Yu, D. Scherer II, R. Camley, Z. Celinski. J. Appl. Phys. 103, 07C508 (2008).

[19] L. Malkinski, M. Yu, A.Y. Vovk, D. Scherer II, L. Spinu, W. Zhou, S. Whittenburg, Z. Davis, J.-S. Jung. J. Appl. Phys. 101, 09J110 (2007).

[20] M.J. Donahue, D.G. Porter. Interagency Report NISTIR 6376. National Institute of Standards and Technology, Gaithersburg; http://math.nist.gov/oommf/

[21] А.Г. Гуревич, Г.А. Мелков. Магнитные колебания и волны. Физматлит, М. (1994). 464 с. 